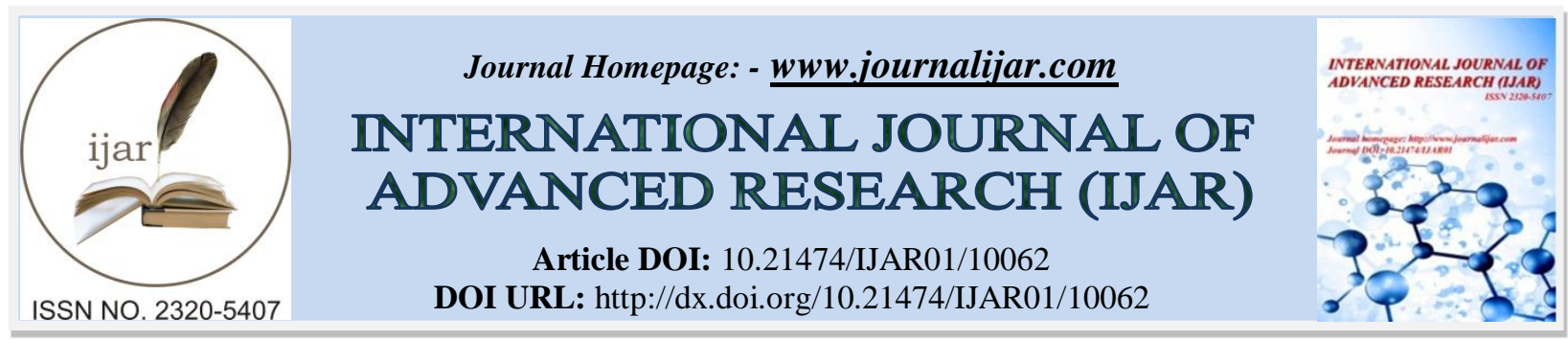

RESEARCH ARTICLE

\title{
ANESTHETIC MANAGEMENT OF A PATIENT WITH CHURG - STRAUSS SYNDROME WHO UNDERWENT REVISION ENDOSCOPIC SINUS SURGERY - CASE REPORT
}

A. Elbouti, A. Jaafari, A. Belghiti, H. Najout, S.D. El Jaouhari and M. Bensghir

Anesthesiology and Reanimation Department, Military Hospital Mohammed V. Rabat

\section{Manuscript Info}

\section{Manuscript History}

Received: 12 September 2019

Final Accepted: 14 October 2019

Published: November 2019

Key words:-

Churg-strauss syndrome, Asthma,

Cholinesterase deficiency, chronic

\section{Abstract}

Churg-strauss syndrome is a rare disease that causes inflammation of small to medium vessels. Diagnostic is mainly clinical with; asthma, eosinophilia and additional vasculitis of multiple organ systems. The anesthesic management of patient with CSS could be complicated by cholinesterase deficiency, hypersensitivity of the airway, chronic steroid usage and multiple organ dysfunctions. Here; we describe our management of a patient with CSS who underwent revision endoscopic sinus surgery under general anesthesia.

The patient had been suffering from asthma for the last 8 years and she was diagnosed with CSS 2 years previously. During the preoperative period; inhaled salbutamol, azathiopirine and oral prednisone were maintained until the operation day, in the morning of surgery she was premedicated with midazolam and hydrocortisone. The anesthesia was induced with propofol, fentanyl and inhaled sevoflurane, any muscle relaxant was not administreted during surgery as there was no need for muscle relaxation for surgery. The surgery was completed uneventfully.

\section{Introduction:-}

Churg strauss syndrome also known as eosinophilic granulomatosis, was described originally in 1951 (1); as a rare disease that causes inflammation of small to medium sized vessels, associated with antineutrophil cytoplasmic antibodies (ANCA). CSS can affect almost any organ system, the commonest site involved is the lung, others organs involved include skin, peripheral and central nervous system, cardio-vascular system, renal system and gastrointestinal tract $(2,3)$.

The exact etiology of CSS is unknown, but immune complexes are probably involved (3). Making diagnostic is difficult because patient with CSS have various clinical symptoms and levels of disease progress; the diagnostic is most commonly based on a combination of specific clinical manifestations that distinguish the syndrome from other primary vasculitidies (Americain Colleage of Rheumatology 1990 criteria) (4). Treatment of patient with CSS is directed toward both immediately reducing the inflammation and suppressing the immune system (cyclophosphamide) (5)

Corresponding Author:-A.Elbouti.

Address:-Anesthesiology and Reanimation department. Military hospital mohammed V. Rabat. 
Patient with CSS display cholinesterase deficiency, airway hyper-reactivity and multi-organ failure (6, 7); for this reasons their anesthesic management could be a real challenge. In the current report we present the anesthesic management of a patient with CSS undergoing revision endoscopic sinus surgery under general anesthesia.

\section{Case report:}

36 year old patient, married and mother of 02 children, in the past history she had been suffering from asthma for the last 8 years, she was currently on the inhaled salbutamol and inhaled symbicort, the last asthma attack goes back more than 6 months. She was diagnosed with CSS 2 years previously when she began to complain of repeated sinusrelated symptoms such as; cheek pain or pressure on both sides and anterior, purulent nasal discharge once or two every month with nasal polyposis ,the chest X-ray showed bilateral interstitial infiltrate, her blood picture showed peripheral eosinophilia and anti-neutrophil cytoplasmic anti-body (ANCA) was negative. The endo-nasal biopsy was performed and demonstrated intense eosinophilic inflammatory infiltrate. A diagnosis of CSS was then made. She had been taking azathiopirine $150 \mathrm{mg} /$ day and prednisone $40 \mathrm{mg} /$ day initially, then progressive reduction of the dose; currently $7.5 \mathrm{mg}$ daily. The patient underwent a functional endo-nasal ethmoidectomy 4 months ago, during which she experienced a severe hemodynamic deterioration related to heavy bleeding with hypotension, and decrease of blood hemoglobin, which required blood transfusion and treatment with vasoactive drugs. The Postoperative was marked by the appearance of a diplopia, exophthalmia and adduction deficit of the left eye. An orthoptic assessment was performed and showed a deficit of the right internal rectus muscle of the left eye, due to internal rectus muscle entrapment. The patient has been programmed for revision endoscopic sinus surgery.

The preoperative physical evaluation showed height and weight of $163 \mathrm{~cm}$ and $86 \mathrm{~kg}$, respectively (BMI of $32 \mathrm{~kg} / \mathrm{m} 2$ ), Lung examination revealed bilateral diffuse wheezing; the cardiovascular, neurological and airway examination were normal. Laboratory studies showed; hemoglobin $10.2 \mathrm{~g} / \mathrm{dl}$, total white blood count (WBC) $8400 / \mathrm{mm}$ with 500/mm eosinophils, kidney function tests along with serum electrolytes were within normal limits. ECG showed sinus rhythm at 72 beats/min with a normal QRS and no ST-T alterations, and the chest x-ray showed bilateral lower lobe minimal infiltrates. Inhaled salbutamol and oral prednisone were maintained until the operation day. In the morning of surgery, the patient was premedicated with iv midazolam $2 \mathrm{mg}$ and hydrocortisone $100 \mathrm{mg}$.

In the operating room; Routine non-invasive monitoring was established, including non-invasive blood pressure (NBP), heart rate $(\mathrm{HR})$, pulse oximetry $\left(\mathrm{SpO}_{2}\right)$, and electrocardiography (ECG). Her baseline blood pressure (BP) and heart rate were $120 / 75 \mathrm{mmHg}$ and $80 / \mathrm{min}$, respectively. Her $\mathrm{SpO}_{2}$ was $99 \%$ while breathing room air and ECG results showed normal sinus rhythm. After obtaining a venous access and 5 minutes of preoxygenation with $100 \%$ oxygen; general anaesthesia was induced with propofol $(200 \mathrm{mg})$ and fentanyl $(250 \mu \mathrm{g})$; after manual ventilation with oxygen-Sevoflurane $3 \%$ for 3 minutes an endotracheal tube (size7) was placed. Anaesthesia was maintained with sevoflurane (MAC 1.5) in air and oxygen (FiO2 50\%), and intermittent boluses of fentanyl $50 \mu \mathrm{g}$. Any muscle relaxant was not administered. The surgery was completed uneventfully as ventilation and blood pressure were adequate throughout the whole operation, no complications occurred and total anesthesia time was $1 \mathrm{~h}$ and 10 minutes, and any anticholinesterase was administered.

At the end of surgery sevoflurane was stopped and tracheal tube was taken out in a deep plane of anesthesia. Oxygen was supplemented by face mask in post anesthesia care unit till patient regained full consciousness.

\section{Discussion:-}

According to the Chapel Hill consensus conference definition Churg Strauss Syndrom is a granulomatous and eosinophil-rich-inflammation involving the respiratory tract, and necrotizing vasculitis affecting small to medium sized vessels and is associated with asthma and eosinophilia $(10,11)$, the diagnostic of CSS is based on a combination of specific clinical manifestation that distingued the syndrome from other primary vasculitidies. Criteria for the classification of CSS were developed by comparing 20 patients who have this diagnostic with 787 control patients with other forms of vasculitis. For the traditionnel classification 6 criteria were selected; Asthma, Eosinophilia >10\% on differential white blood cell count, mononeuropathy (including multiplex) or polyneuropathy, non fixed pulmonary infiltrate on X-ray, paranasal sinus abnormality and biopsy containing a blood vessels with extra vascular eosinophils. The presence of 4 or more of these 6 criteria yielded a sensitivity of $85 \%$ and a specificity of $99,7 \%$. A classification tree was also constructed with 3 selected criteria; asthma, eosinophilia > $10 \%$ on differential white blood cell count and history of documented allergy other than asthma or allergy (ACR 1990) (4). Our patient's asthma, pulmonary infiltrate on x-ray, peripheral blood eosinophilia, nasal polyposis, and the endo-nasal biopsy with eosinophilic inflammatory infiltrate. 
Its incidence is variably reported from 2, 4 to 40 cases per one million people it can affect all age groups with the average of 35 to 45 years. Also, male are more commonly affected than females (12).

CSS treatment is based on use of high dose corticosteroids and other immunosuppressive agents (7artII), prednisone is the most commonly prescribed drug; high dose of prednisone are initially used (40-60 mg /day), after the first month or so, this high dose of prednisone is slowly tapered down over the ensuing months (5mg every one to two weeks) $(6,13)$. Immunosuppressive drug such as cyclophosphamide, azathiopirine or methotrexate may be used in addition to prednisone to reduce the body's immunoreaction; induction can occur with cyclophosphamide and the maintenance with azathiopirine (5). Other biological drugs can also be used such rituximab or milolizumab (5). The French vasculitis study group developed five prognostic factors (five factor score: FFS) that predict death for patient with eosinophilic granulomatosis with polyangiitis including CSS (table 1)

Table 1:-Five factor score for patient with eosinophilic granulomatosis with polyangiitis (EGPA- CSS) (5)

\section{Factors}

- $\quad$ Reduced renal fun ction (creatinine $>1,58 \mathrm{mg} / \mathrm{dl}$ or $140 \mathrm{mmol} / \mathrm{l}$ )

- $\quad$ Proteinuria ( $>1 \mathrm{~g} / 24$ hours)

- Cardiomyopathy

- Central nervous system involvement

- Gastro-intestinal manifestation (GI hemorrhage, GI infarction, or pancreatitis)

\begin{tabular}{|l|l|}
\hline FFS: five factor score & \multicolumn{1}{|c|}{ Five year mortality rate \% } \\
\hline $\begin{array}{l}\text { FFS }=0 \\
\text { these factors }\end{array}$ & \\
\hline FFS $=1:$ Severe disease ; presence of one factor & $26 \%$ \\
\hline FFS $\geq 2:$ very severe disease, two factors or & $46 \%$ \\
more & \\
\hline
\end{tabular}

In pre-operative evaluation of patients with CSS, pulmonary function tests (PFTs) in symptomatic patients are necessary to predict peri-operative and post operative pulmonary complications, our patient had been diagnosed with asthma for the last 8 years, but her asthma is usually well controlled with inhaled salbutamol and inhaled symbicort and the last asthma attack goes back more than 6 months. Cardiac involvement is known to be the major cause of death in CSS; therefore, the assessment of cardiac status should be performed to rule out eosinophilic endomyocardities, coronary dissection, coronary vasculitis, conduction abnormalities, congestive heart failure and pericardial effusion (12).

Regional anesthesia should be preferred because patients with asthma who require general anesthesia and tracheal intubation are considered to be at increased risk for the development of bronchospasm during anesthesia. However; in Warner's study for over 1550 patients with asthma, the complication rates for general and regional anesthesia were similar refuting the notion that regional anesthesia was safer for patients with asthma $(2,14)$. Patient should be not eat or drink for 6 hours before surgery with an exception being medication; patients with CSS and who have been taking steroids therapy for a long time may develop secondary adrenalis insufficiency that can manifest as full blown adrenalin crisis in the peri operative period, CSS patients should take their usual medication up until the time of surgery and may require peri operative stress dose steroids to mitigate this rare but potentially fatal complication of chronic steroid use $(6,15)$. Our patient took inhaled salbutamol and oral prednisone on the morning of surgery, and Hydrocortisone $100 \mathrm{mg}$ was given intravenously before induction.

The anesthesic management has been focused on avoiding bronchoconstriction and inducing bronchodilatation. Propofol and ketamine inhibit bronchoconstriction decreasing the risk of bronchospasm during anesthesia induction; propofol yields central airway and is more reliable than etomidate or thiopental (16). Volatile anesthesic except desflurane are potent bronchodilatateur decrease airway responsiveness and attenuate histamine induced bronchospasm. Opioilds could release histamine; however, they are considered safe for patients with increased bronchial reactivity, fentanyl and its analogues are frequently used in the induction of anesthesia, and they can lead to thorax rigidity that can be misinterpreted as bronchospasm, with slow injection this effect is hardly observed. 
Moreover, the suppression of the cough reflex and deeping of anesthesia level achieved after Opioild administration can be helpful in asthmatic patients. (16)

Neuromuscular blocking drug with minimal histamine release such as rocuronium, cisatracurium, vecuronium and pancuronium can be used in patients with CSS ; Taylor and colleagues reported two patients with CSS who were found to have cholinesterase deficiency, both patients received immunosuppressive therapy, but differenciation between the effects of immunosuppressive and the disease process itself cannot be established (17). Furthmore, without information regarding patient's plasma cholinesterase level or activity; depolarizing neuromuscular blocker (Succinylcholine) should be used carefully and if necessary in reduced dosage (18).

However; few case reports described caution or complete avoidance of non depolarizing muscle relaxant to avoid using anticholinesterase agent at the end of surgery as reversals which can cause bradycardia, increased secretions and bronchial hyperreactivity. Hyo Sang Im et al (6) reported a case of patient with CSS who underwent endoscopic sinus surgery under general anesthesia; they used propofol, sevofluane and remifentanyl for induction, and anesthesia was maintained with sevoflurane and remifentanyl, they did not use any muscle relaxant. Gurjar M et al (2); also described the anesthesic considerations in a patient with the CSS undergoing radical mastectomy, the anesthesia was induced with propofol and maintained by isoflurane and iv propofol infusion along, without paralyzing the patient, and the airway was secured with laryngeal mask. For our case, the anesthesia management was successful; we used propofol, fentanyl and sevoflurane for induction and continued sevoflurane and intermittent boluses of fentanyl as maintenance agents. Any muscle relaxant was not adminestreted as there was no need for muscle relaxation for surgery, therefore; no anticholinesterase was administered.

But this strategy may not always be practical; Ping el al (18), described anesthesic management of a patient with CSS undergoing abdominal hysterectomy, which required deep paralysis for optimal operating conditions; the anesthesia was induced with propofol, ketamine, fentanyl and rocuronium, and maintened wth sevoflurane, during surgery, further rocuronium bolus was given for adequate paralysis, at the end of surgery they used sugammadex for reversal of neuromuscular blockade. So, if a patient requires a rapid sequence induction but abnormal sensitivity to suxamethonium is to be avoided, rocuronium can be considered the paralytic agent of choice, and sugammadex can be successfully used for reversal of neuromuscular blockade (18)

In conclusion, CSS is a rare disease that can affect various organ systems, unfortunately; there are only few studies describing anesthetic management in CSS patients. This case was successfully managed following an anesthesia plan taking into consideration; airway hyperreactivity, cholinesterase deficiency and chronic corticosteroid usage.

\section{References:-}

1. Churg J, Strauss L. Allergic granulomatosis, allergic angiitis and periarteritis nodosa. Am J Pathol 1951; 27(2): 277-301.

2. Gurjar M, Bhatnagar S, Mishra S, Jain V, Singhal AK. A case of Churg-Strauss syndrome undergoing radical mastectomy under general anaesthesia and thoracic epidural analgesia. Eur $\mathrm{J}$ Anaesthesiol. 2006 Nov;23(11):980-2.

3. Fasciolo A, Inglese S, Farina P, Baldini C. Videoendoscopic nasal polypectomy in Churg-Strauss syndrome: anaesthesiological management. Internet J Anesthesiol. 2007;18(2):1-6.

4. Masi AT, Hunder GG, Lie JT, Michel BA, Bloch DA, Arend WP, Calabrese LH, Edworthy SM, Fauci AS, Leavitt RY, et al. The American College of Rheumatology 1990 criteria for the classification of ChurgStrauss syndrome (allergic granulomatosis and angiitis). Arthritis Rheum. 1990 ;33(8):1094-100.

5. Gregoire-Bottex MM, Greydanus DE, Cates KW. Eosinophilic granulomatosis with polyangiitis (EGPA): an update for the Churg-Strauss syndrome. EC Pulmonol Respirat Med. 2017;4(4):132-8.

6. Hyo Sang Im, Kwang-Rae Cho. A patient with Churg-Strauss syndrome who underwent endoscopic sinus surgery under general anesthesia. Korean J Anesthesiol. 2010 Jul; 59(1): 49-52.

7. Preeti V, Paul AG. Churg-Strauss syndrome (Survival for 26 years). Annals of allergy asthma and immunology. 2002; 88(6): 640-43.

8. Kurita M, Niwa Y, et al. Churg-Strauss syndrome (allergic granulomatous angiitis) with multiple perforating ulcers of the small intestine, multiple ulcers of the colon, and mononeuritis multiplex. J Gastroenterol. 1994 Apr;29(2):208-13

9. Hall FC, Walport MJ. Hypereosinophilic syndromes: association with vasculitis, fibrosis and autoimmunity. Clin Exp Allergy. 1993 Jul; 23(7):542-7. 
10. Siemińska A. [Churg-Strauss syndrome--a rare disease or a difficult diagnosis?]. Pneumonol Alergol Pol. 2012; 80(1):3-5.

11. Jennette JC, Falk RJ. Nomenclature of systemic vasculitides. Proposal of an international consensus conference. Arthritis Rheum. 1994 Feb; 37(2):187-92.

12. Masoud Nashibi, Ardeshir Tajbakhsh. Anesthetic Management of T12 Schwannoma in a Patient with ChurgStrauss Syndrome: A Case Report. Arch Neurosci. 2018 October; 5(4):e81059

13. Pagnoux C1, Guilpain P, Guillevin L. Churg-Strauss syndrome. Curr Opin Rheumatol. 2007 Jan; 19(1):25-32.

14. Warner DO, Warner MA. Perioperative respiratory complications in patients with asthma. Anesthesiology. 1996 Sep;85(3):460-7.

15. Liu MM, Reidy AB. Perioperative Steroid Management: Approaches Based on Current Evidence. Anesthesiology. 2017 Jul;127(1):166-172.

16. Burburan SM1, Xisto DG. Anaesthetic management in asthma. Minerva Anestesiol. 2007 Jun; 73(6):357-65.

17. Taylor BL, Whittaker M, Van Heerden V, Britten J. Cholinesterase defi- ciency and the Churg-Strauss syndrome. Anaesthesia. 1990;45(8):649- 52

18. Ping Han Chia. Anaesthetic management and the role of sugammadex in a patient with Churg-Strauss syndrome. Indian J Anaesth. 2018 May; 62(5): 400-402. 